\title{
Statistics of Attenuation Due to Rain Affecting Hybrid FSO/RF Link: Application for 5G Networks
}

\author{
U. A. Korai ${ }^{1,2}$, L. Luini ${ }^{3,4}$, R. Nebuloni ${ }^{4}$, I. Glesk ${ }^{1}$ \\ ${ }^{1}$ Department of Electrical and Electronics Engineering, University of Strathclyde, Glasgow, United Kingdom \\ ${ }^{2}$ IICT, Mehran University of Engineering and Technology, Jamshoro, Pakistan \\ ${ }^{3}$ DEIB, Politecnico di Milano, Milan, Italy \\ ${ }^{4}$ IEIIT, Consiglio Nazionale delle Ricerche (CNR), Milan, Italy
}

\begin{abstract}
This paper shows that FSO could be an option for backhauling among microcells in future $5 \mathrm{G}$ systems in areas where fog is not a concern as very short LOS links (few hundred meters) would be required. In this respect, monthly CCDFs of rain attenuation at both $R F(28 \mathrm{GHz})$ and optical bandwidth have been calculated in the area of Hyderabad (Pakistan). FSO links with simple OOK modulation would be able to reach targeted data rate of hundreds of Gbps for $\mathbf{9 9 . 9 9 \%}$ of time provided the link length is less than few hundreds of meters.
\end{abstract}

Index Terms - Free Space Optics, rainfall effects, electromagnetic wave propagation.

\section{INTRODUCTION}

The future of terrestrial wireless telecommunication technologies mainly depends on high carrier frequencies (above $10 \mathrm{GHz}$ ) and these technologies typically require Line Of Sight (LOS) transmission. One of the most preferable LOS technologies is wireless optical communication, often known as Free Space Optics (FSO). FSO works in the optical bandwidth (i.e. hundreds of $\mathrm{THz}$ ) and it can provide data rates in the order of hundreds of Gbps [1]. Besides very high capacity, FSO provides several attractive features if compared with Radio Frequency (RF) and microwaves, such as high directivity, quick deployment, easy installation, unlicensed frequency band and virtually no electromagnetic compatibility issues. On the other hand, the main problem associated to FSO is the impact of weather on the system availability and Quality of Service (QoS). The stronger effect comes from fog, which can attenuate the FSO signal with fade levels in excess of $300 \mathrm{~dB} / \mathrm{km}$ [2], [3]. Where fog is not an issue, rain represents the main impairment to terrestrial FSO systems, and it is able to severely limit their coverage.

As a result, FSO is typically considered for short-distance communications (say up to $2 \mathrm{~km}$ ). For example, FSO links may be used to interconnect two Base Transceiver Stations (BTSs) in future $5 \mathrm{G}$ networks. As Rappaport et al. suggest in [4], the typical expected cell size is in the order of $200 \mathrm{~m}$, and the target frequencies for a RF system are $28 \mathrm{GHz}$ and $38 \mathrm{GHz}$. The aim of this contribution is to investigate the use of hybrid $\mathrm{FSO} / \mathrm{RF}$ links to interconnect BTSs in $5 \mathrm{G}$ mobile networks, the main focus being the impact of precipitation on link. As for RF, we consider $28 \mathrm{GHz}$ thanks to the lower fade levels if compared to $38 \mathrm{GHz}$. Specifically, we investigate the changes in specific attenuation $\gamma$ associated to different Drop Size Distributions (DSDs), in turn modeled using the Gamma function [5].

As part of this analysis, a method is proposed to estimate the statistics of rain attenuation affecting a short FSO terrestrial link and an RF link, using as input local rain rate statistics with 1-minute integration time. In this work, such statistics, henceforth referred to as $P(R)$, are obtained from the global model presented in [6], which only requires as input the average value of the rain cumulated in a year or in a month. In this work, we have selected one site, i.e. Hyderabad, Pakistan, and considered 3 months (July, August and September), for which the rainfall accumulation is higher.

\section{GAMMA DSD AND RAIN ATTENUATION FOR FSO AND RF LINKS}

Information on the DSD is very important in order to obtain accurate estimates of the rain attenuation affecting FSO links. In this work, we have considered the Gamma model for the DSD, which has the following expression:

$$
\mathrm{N}(\mathrm{D})=\mathrm{N}_{0} \mathrm{D}^{\mu} \exp (-\Lambda \mathrm{D})
$$

In (1), $N(D)$ is the number of drops per unit volume of diameter included between $D$ and $D+\Delta D$ (in mm) divided by $\Delta D$ and it is measured in $\mathrm{m}^{-3} \mathrm{~mm}^{-1}, N_{0}$ is the intercept parameter, measured in $\mathrm{mm}^{-1-\mu} \mathrm{m}^{-3}, \Lambda$ is the slope parameter measured in $\mathrm{mm}^{-1}$, and $\mu$ is the shape parameter. Measurements show that $\mu$ typically ranges between -3 and 8 [5]. According to [8], it is possible to relate both $N_{0}$ and $\Lambda$ to $\mu$, such that the latter turns out to be the only parameter driving the shape of (1):

$$
\begin{gathered}
N_{0}=6 \times 10^{3} e^{(3.2-\ln 5) \mu-\ln 5} \\
\Lambda=0.2\left[\frac{R}{33.31 N_{n} 5^{1+\mu} \Gamma(4.67+\mu)}\right]^{-\frac{1}{4.67+\mu}}
\end{gathered}
$$

where $R$ is the rain rate $(\mathrm{mm} / \mathrm{h})$. The specific attenuation $\gamma$ (in $\mathrm{dB} / \mathrm{km}$ ) due to rain can be calculated as: 


$$
\gamma=4.343 \times 10^{-3} \int_{D_{1}}^{D_{2}} C_{e x t}(\lambda, D) N(D) d D
$$

where, $D_{1}$ and $D_{2}$ are the limits of the drop size (mm), i.e. the minimum and maximum drop diameter, respectively; $C_{\text {ext }}(\lambda, D)$ is the extinction cross-section of the particle (assumed spherical for sake of simplicity) and measured in $\mathrm{mm}^{2}$. In this work, we calculated $C_{\text {ext }}$ by using the asymptotic theory mentioned in [7], which states that if the size of the particle is much larger than the wavelength (as in case of the raindrops in the optical region), the extinction cross section is twice as the geometrical cross section of the particle, i.e., assuming spherical raindrops:

$$
C_{e x t}=2 \pi D^{2}
$$

Though it is well known that large raindrops tend to take the form of oblate spheroids, the assumption of equivolume spherical particles does not produce significant errors when a statistical characterization of rain attenuation is carried out and wave polarization effects are not considered.

In this work, we have specifically consider a single site, i.e. Hyderabad, Pakistan, for which, the precipitation intensity is fairly limited. As a result, we have selected only three DSDs, namely $\mu=1,2$ and 3. Moreover, the relationship between $R$ and $\gamma$, calculated according to (4), is fitted using the customary power-law relationship in (7):

$$
\gamma=k R^{\infty}
$$

where $k$ and $\alpha$ are the fitting parameters. Table I shows parameters for possible values of $\mu$ for FSO. The fitted parameters, $k$ and $\alpha$, for the RF link depends of frequency and microstructure of rain and can be calculated using ITU$\mathrm{R}$ terrestrial model [9]. Table II also includes the parameter values for $28 \mathrm{GHz}$ and $38 \mathrm{GHz}$ frequency.

TABLE I. FITTING COEFFICIENTS IN (7) FOR FSO LINK FOR DIFFERENT SHAPE PARAMETERS

\begin{tabular}{|c|c|c|}
\hline $\begin{array}{c}\text { Shape parameter } \\
(\boldsymbol{\mu})\end{array}$ & $\boldsymbol{k}$ & $\alpha$ \\
\hline 1 & 1.1399 & 0.7056 \\
\hline 2 & 1.0506 & 0.7497 \\
\hline 3 & 0.9939 & 0.7823 \\
\hline
\end{tabular}

TABLE II. FITTING COEFFICIENTS IN (7) FOR RF LINK FOR 28GHZ AND $38 \mathrm{GHz}$

\begin{tabular}{|c|c|c|}
\hline $\begin{array}{c}\text { Shape parameter } \\
(\boldsymbol{\mu})\end{array}$ & $\boldsymbol{k}$ & $\alpha$ \\
\hline $28 \mathrm{GHz}$ & 0.2051 & 0.9679 \\
\hline $38 \mathrm{GHz}$ & 0.4001 & 0.8816 \\
\hline
\end{tabular}

Fig. 1 shows the specific attenuation as a function of rain rate obtained using (7) and the parameters listed in Table I and Table II. Fig. 1 clearly shows that the specific attenuation affecting RF links is lower than the one impairing FSO links. Therefore, it is a good option to use the
RF link as a backup when the FSO link goes into outage due to precipitation. Specifically, for the link performance predictions included in the remainder of this work, we have chosen $28 \mathrm{GHz}$ link for the RF link and $\mu=1$ for FSO link.

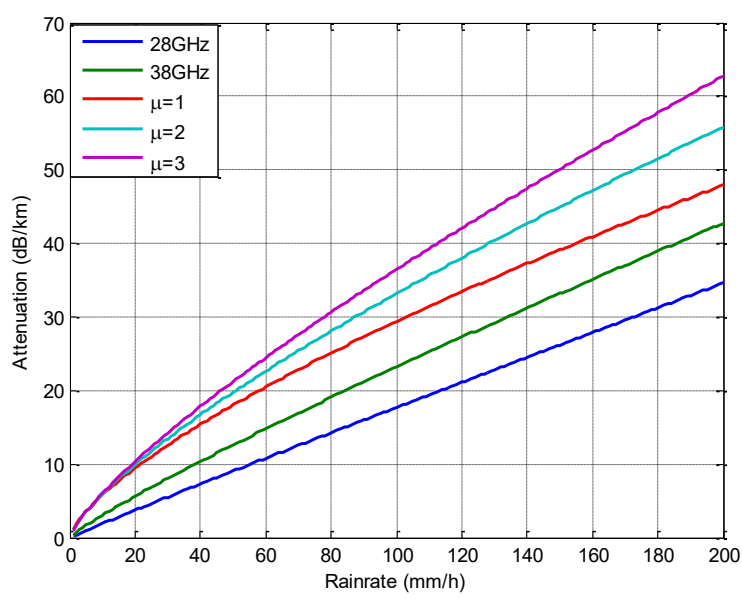

Fig. 1. Specific attenuation $(\mathrm{dB} / \mathrm{km})$ as a function of rain rate $(\mathrm{mm} / \mathrm{h})$ for FSO and RF link.

\section{MONTHLY RAIN RATE STATISTICS}

In order to investigate the performance of FSO and RF link in presence of rain, local precipitation data are required. Specifically, for system performance analysis, the information required is the Cumulative Distribution Function of the rain rate with 1-minute integration time, also referred to as $P(R)$. As this is not easily retrievable worldwide, various techniques have been developed to estimate it, such as integration time conversion models [13] and models based on meteorological maps [14]. In this work, we use MORSE [10] to estimate local $P(R)$ s on monthly basis, starting only from monthly rainfall accumulations $M_{t}$, collected for twenty years at Hyderabad, Pakistan. Only the months with the highest monthly accumulation values (see Table III, rain gauge data provided by the Pakistan meteorological department) were used to derive the local $P(R)$, which, according to MORSE, is given by:

$$
P(R)=P_{0}\left[\ln \left(\frac{R_{a}+R_{\text {low }}}{R+R_{\text {low }}}\right)\right]^{n}
$$

In (8), $R$ is the rain rate $(\mathrm{mm} / \mathrm{h})$ exceeded with probability $P, P_{0}$ defines the behavior of the curve for $R \rightarrow 0$ $\mathrm{mm} / \mathrm{h}, R_{a}$ is the asymptotic value of $R, n$ mainly determines the shape of the curve and $R_{\text {low }}$ allows the probability to assume a finite value when $R \rightarrow 0 \mathrm{~mm} / \mathrm{h}$.

The parameters $P_{0}, n, R_{a}$ and $R_{l o w}$ are related to $M_{t}$ and $\beta$ : here the former is obtained from the rain gauge average levels in Table III (last row), whereas the latter, named convectivity ratio, is extracted from the ERA40 database which MORSE relies on [10]. The parameters $P_{0}, n, R_{a}$ and $R_{\text {low }}$ can be calculated as: 


$$
\begin{gathered}
n=36.18 B^{0.1242}+36.92 \\
n=8.43 \times 10^{-4} R_{a}^{1.3531}+1.44 \\
R_{\text {low }}=\left\{\begin{array}{cc}
31.82 B^{-0.0086}-31.94 & B \leq 0.72 \\
10^{-4} & B>0.72
\end{array}\right.
\end{gathered}
$$

TABLE III. WORST MONTHLY RAIN DATA FOR THE REGION OF HYDERABAD, PAKISTAN FOR 20 YEARS.

\begin{tabular}{|c|c|c|c|c|}
\hline $\begin{array}{c}\text { Number } \\
\text { of years }\end{array}$ & Year & $\begin{array}{c}\text { Annual } \\
\text { cumulated } \\
\text { rain for July } \\
\text { (mm) }\end{array}$ & $\begin{array}{c}\text { Annual } \\
\text { cumulated } \\
\text { rain for } \\
\text { August (mm) }\end{array}$ & $\begin{array}{c}\text { Annual } \\
\text { cumulated } \\
\text { rain for } \\
\text { September } \\
\text { (mm) }\end{array}$ \\
\hline 1 & 1995 & 78.6000 & 0.8000 & 0.1000 \\
\hline 2 & 1996 & 6.1000 & 0.1000 & 0 \\
\hline 3 & 1997 & 0.8000 & 9.0000 & 0 \\
\hline 4 & 1998 & 8.6000 & 0.1000 & 8.4000 \\
\hline 5 & 1999 & 0.7000 & 0.1000 & 0 \\
\hline 6 & 2000 & 8.0000 & 46.6000 & 0 \\
\hline 7 & 2001 & 104.9000 & 47.5000 & 0.1000 \\
\hline 8 & 2002 & 0 & 0.1000 & 0 \\
\hline 9 & 2003 & 9.2000 & 77.4000 & 0 \\
\hline 10 & 2004 & 0 & 16.0000 & 0.1000 \\
\hline 11 & 2005 & 10.6000 & 15.4000 & 3.2000 \\
\hline 12 & 2006 & 80.2000 & 4.5000 & 170.0000 \\
\hline 13 & 2007 & 7.5000 & 130.4000 & 0 \\
\hline 14 & 2008 & 8.0000 & 103.8000 & 0 \\
\hline 15 & 2009 & 137.8000 & 62.2000 & 0 \\
\hline 16 & 2010 & 29.5000 & 85.9000 & 18.0000 \\
\hline 17 & 2011 & 4.1000 & 10.4000 & 56.9000 \\
\hline 18 & 2012 & 3.2000 & 2.2000 & 96.2000 \\
\hline 19 & 2013 & 2.5000 & 61.8000 & 12.0000 \\
\hline 20 & 2014 & 16.0000 & 1.2000 & 0 \\
\hline Average (1995-2014) & 25.8150 & 33.7750 & 18.2500 \\
\hline & & & & \\
\hline
\end{tabular}

Fig. 2 shows the 1-minute rain statistics of the worst months estimated using MORSE for Hyderabad, Pakistan.

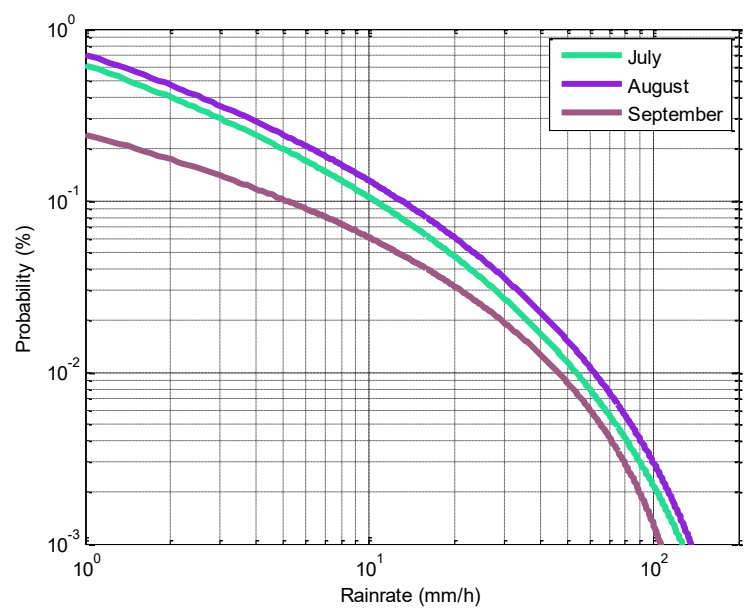

Fig. 2. Monthly 1-min integrated $P(R) \mathrm{s}$ for Hyderabad, Pakistan, as derived from MORSE using as input the mean yearly $M_{t}$ value measured locally.

\section{RAIN ATTENUATION STATISTICS}

In [11], we have calculated the overall yearly attenuation starting from the yearly rainfall accumulations recorded by the rain gauge. In this contribution, we address monthly statistics, specifically those months for which the impact of rain is stronger. As for specific attenuation, the reason for choosing $\mu=1,2$ and 3 is that the region of Hyderabad lies in between arid and semi-arid climatic zones [12], in which the overall probability to have rain is fairly limited and is typically convective. Compared to stratiform rain, convective precipitation is characterized by an increased number of large drops, which is reflected by the Gamma distribution in (1) with $\mu=1,2$ and 3 . by:

The total attenuation, $A$, for a link of length $L$, is given

$$
A=\int_{L} \gamma(l) d l
$$

In the clear weather, FSO link is considered to be the main link for interconnecting the BTSs as it provides 100s of Gbps data rate henceforth connect many users as compared to the RF link but in presence of the rain, which disturbs the targeted availability of the FSO system, 99.99\%, the main link will be shifted to the RF link as this link is less attenuated as compared to the FSO link, as shown in Fig. 1. The switching of the link from FSO to RF will decrease the bandwidth, henceforth the data rates. Therefore, few of the users may not be able to get connected when the link is shifted from FSO to RF but the availability will be maintained, which is the goal of this contribution

Since we are considering the short distance link therefore, the specific attenuation can be assumed to be constant along the whole link $(L)$. As a consequence, equation (12) becomes:

$$
A=\gamma \times L
$$

Fig. 3 and Fig. 4 depict the Complementary Cumulative Distribution Function (CCDF) of rain attenuation in Hyderabad for $L$ up to $1 \mathrm{~km}$, using as input the $P(R)$ associated to August. Table IV summarized the power margins required to counteract rain attenuation and achieve link availability of $99.99 \%$ 


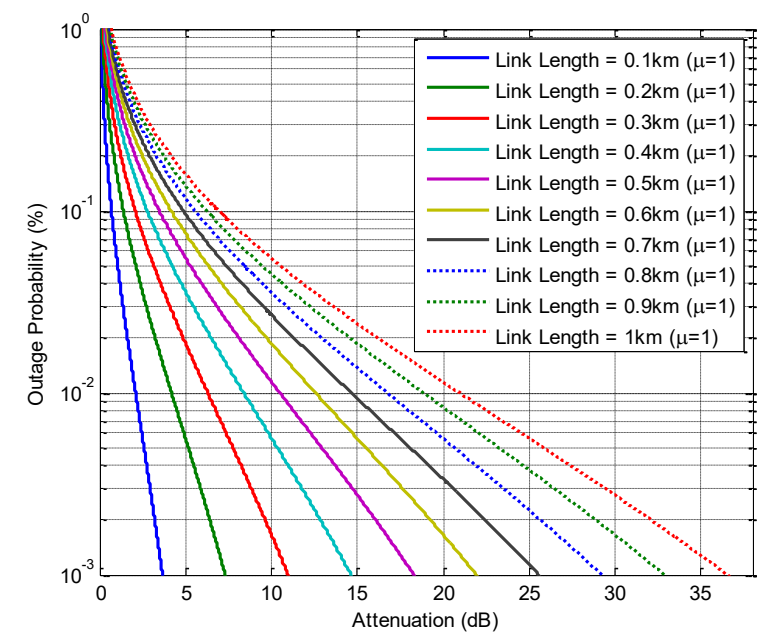

Fig. 3. CCDF of rain attenuation for FSO link $(\mu=1)$ for various values of $L$ (up to $1 \mathrm{~km})$.

TABLE IV. 99.99\% AVAILABILITY MARGIN REQUIRED TO COPE WITH RAIN ATTENUATION IN HYDERBAD (AUGUST)

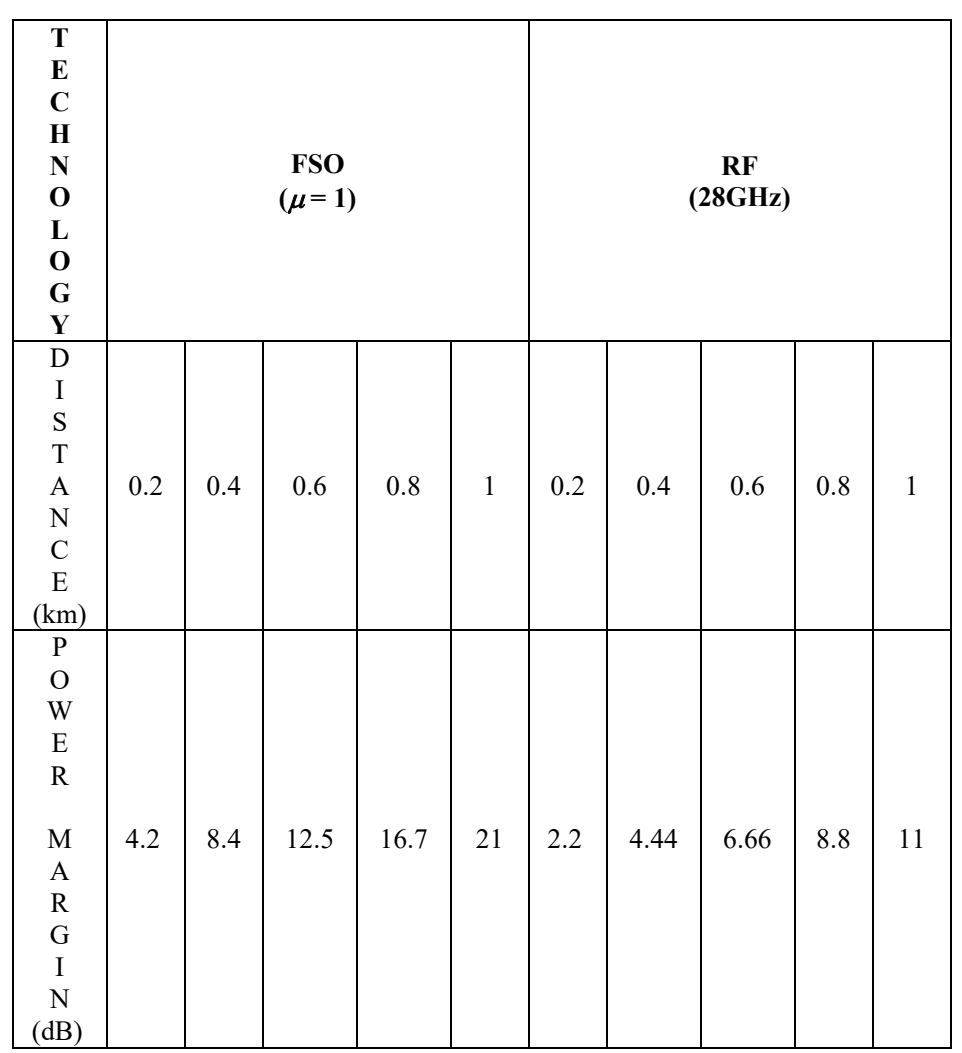

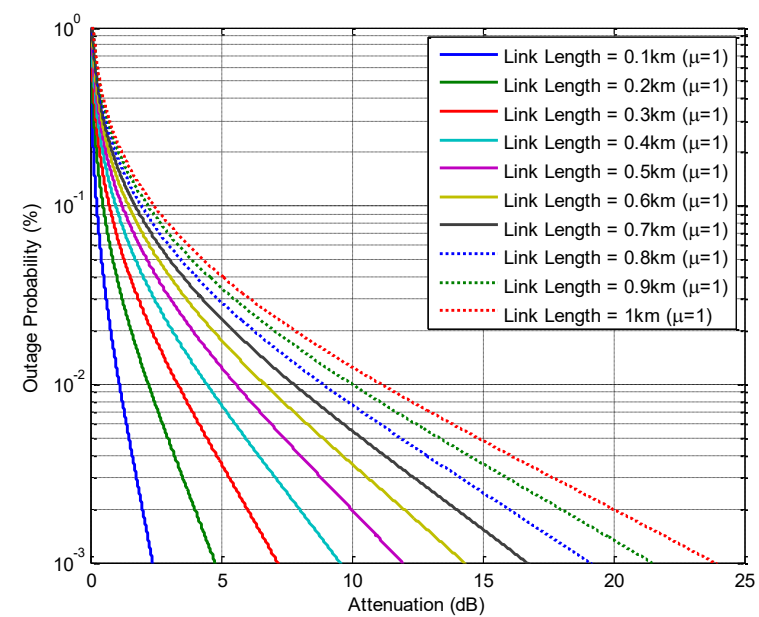

Fig. 4. CCDF of rain attenuation at $28 \mathrm{GHz}$ for various values of $L$ (up to $1 \mathrm{~km})$.

\section{ONCLUSION}

In this contribution, we described to calculate the total attenuation using 1-minute rain integrated data for FSO, RF and hybrid FSO/RF link. The 1-minute rain data is computed using MORSE by inputting the monthly data. We have considered the August month as a worst month as the accumulated rain rate per month for the August is high as compared to the other months. For the rain data, we have considered the 20 years monthly rain data of Hyderabad, Pakistan. The main idea of this work is to use the FSO as a main link for the $5 \mathrm{G}$ networks. The use of FSO link gives ease of communication and fulfils the demands of future $5 \mathrm{G}$ users because of its very high bandwidth feature but in presence of rain the targeted availability, $99.99 \%$, of the FSO system is turned down. Since the attenuation due to rain for the FSO system is quite high in contrast to the RF link and therefore, to maintain the targeted availability, the link is shifted from FSO to RF. It can be seen from the results that to achieve the targeted system availability at the distance of $1 \mathrm{~km}$, RF link uses less than $12 \mathrm{~dB}$ of power margin in contrast to the FSO technology, i.e. $21 \mathrm{~dB}$, keeping all other parameters constant, but this shift will reduce the data rates and will accommodate less number of users as compared to the FSO.

\section{ACKNOWLEDGMENT}

The authors would like to thank Mr. Abdul Qayoom Bhutto, Director Pakistan Meteorological Department, and $\mathrm{Mr}$. Nadeem Faisal who help us to provide the Hyderabad, Pakistan rain data for 20 years.

\section{REFERENCES}

[1] W. S. Tsai, H. H. Lu, C. Y. Li, T. C. Lu, H. H. Lin, B. R. Chen, C. J. Wu, "A 50-m/320-Gb/s DWDM FSO Communication With Afocal Scheme,” IEEE Photonics Journal, Volume: 8, Issue: 3, 2016.

[2] L. Luini, C. Capsoni, and R. Nebuloni, "Free space optics to enable high data rate download from leo satellites: the impact of clouds," 3rd 
International Workshop in Optical Wireless Communications (IWOW), pp. 35-39, 2014

[3] F. Nadeem, V. Kvicera, M. S. Awan, E. Leitgeb, S. S. Muhammad, G. Kandus, "Weather Effects on Hybrid FSO/RF Communication Link," IEEE Journal on Selected Areas in Communications, Vol. 27, No. 9, pp. 1687-1697, 2009

[4] T. S. Rappaport, S. Sun, R. Mayzus, H. Zhao, Y. Azar, K. Wang, G. N. Wong, J. K. Schulz, M. Samimi, F. Gutierrez, "Millimeter wave mobile communications for 5G cellular: it will work!," IEEE Access, pp. 335-349, 2013

[5] C. W. Ulbrich, "Natural variations in the analytical form of the raindrop size distribution," J. Climate Appl. Meteor., 22, 1764-1775, 1983

[6] L. Luini, C. Capsoni, "A unified model for the prediction of spatial and temporal rainfall rate statistics," IEEE Transactions on Antennas and Propagation, Vol. 61, No. 10, pp. 5249-5254, October 2013

[7] W.J. Wiscombe, "Improved Mie scattering algorithms," Appl. Opt. 19, pp. 1505-1509, 1980

[8] D. Atlas, R. C. Srivastava, R. S. Sekhon, "Doppler radar characteristics of precipitation at vertical incidence," Rev Geophys Space Phys, 2, pp. $1-35,1973$

[9] ITU-R, P.838-3: "Specific Attenuation Model for Rain for use in Prediction Methods".

[10] L. Luini, C. Capsoni, "A unified model for the prediction of spatial and temporal rainfall rate statistics," IEEE Transactions on Antennas and Propagation, Vol. 61, No. 10, pp. 5249-5254, October 2013

[11] U. A. Korai, L. Luini, R. Nebuloni, C. Capsoni, K. Dev, "Modeling Statistics of Rain Attenuation Affecting FSO links: a Case Study," $10^{\text {th }}$ European Conference on Antenna and Propagation, April 2016, IEEE

[12] M. Zahid, G. Rasul, "Rise in Summer Heat Index over Pakistan," Pakistan Journal of Meteorology, Vol. 6, Issue 12, pp. 85-96, 2010.

[13] L.D. Emiliani, L. Luini, C. Capsoni, "Extension of the ITU-R method for the conversion of rain rate statistics from various integration times to one minute," Electronics Letters, Vol. 44, Issue 8, pp. 557-558, 10 April 2008.

[14] Characteristics of precipitation for propagation modelling. Geneva, 2012, ITU-R recommendation P.837-6 